\title{
Clash of the microbes: let's bring back the good guys
}

\author{
Martin J. Wolff, MD, ${ }^{1}$ Michael A. Poles, MD, ${ }^{1}$ and Judith A. Aberg, MD ${ }^{2}$
}

${ }^{1}$ Division of Gastroenterology and ${ }^{2}$ Division of Infectious Diseases and Immunology, Department of Medicine, New York University School of Medicine, New York, New York, USA.

\begin{abstract}
A 38-year-old man with a history of HIV infection virologically suppressed on antiretroviral therapy presents to his gastroenterologist for evaluation of iron deficiency anemia and weight loss. A diagnostic colonoscopy demonstrates a two-centimeter ulcerated mass in the cecum. Biopsies of the lesion return moderately differentiated adenocarcinoma that is wild type for the KRAS mutation by real-time PCR.
\end{abstract}

It is truly fascinating that we are only $10 \%$ human, sorely outnumbered by the intestinal bacteria we harbor within (1). Our incredibly complex microbiome is functionally an organ unto itself, weighing up to $1 \mathrm{~kg}$ and serving as a "virtual bioreactor" that facilitates digestion, nutrient absorption, and the trajectory of our immune system (1). Intuitively, alterations in the composition of our intestinal symbionts may have as much impact on our health as other major medical interventions.

There has been an explosion of interest in probiotics over the last decade. On the shelves of supermarkets and pharmacies nationwide, this markedly heterogeneous class of medications promises improved colonic health, digestive regularity, and overall well-being. Mechanistically, probiotics are believed to modulate intestinal immune function by increasing secretory IgA, reducing proinflammatory cytokine production, enhancing mucosal barrier function, and reducing bacterial attachment (1). There is mounting evidence to support the use of various probiotics for infectious and Clostridium difficile-associated diarrhea, irritable bowel syndrome, inflammatory bowel disease and post-surgical complications thereof (i.e. pouchitis), and hepatic encephalopathy (1).

Emerging studies lend credence to the concept that modulation of intestinal flora can provide benefit in HIV infection. Within days following infection, there is a rapid and sustained depletion of gut mucosal $\mathrm{CD}^{+} \mathrm{T}$ cells in HIV-

Conflict of interest: The authors have declared that no conflict of interest exists.

Citation for this article: J Clin Invest. 2013; 123(2):544-545. doi:10.1172/JCI66736. positive individuals (ref. 2 and Figure 1). In particular, there is a massacre of gastrointestinal-associated (GI-associated) $\mathrm{CD}^{+}$Th17 cells, which differentiate in response to normal bacterial microflora (3). This depletion, combined with local immune activation, is thought to directly contribute to defective mucosal barrier function, increased intestinal permeability, augmented translocation of intestinal microbial products, and to the vulnerability of opportunistic infection (4). Importantly, antiretroviral therapy (ART) does not readily reverse these deficits in the GI mucosa, which can persist despite reconstitution of the peripheral $\mathrm{CD} 4^{+}$ repertoire (5). It has been hypothesized that systemic immune activation may be reduced by enhancing the mucosal barrier, decreasing the degree of translocated microbial products, or both. The latter is being addressed in a clinical investigation of rifaximin in HIV-infected subjects (NCT01466595). Probiotics may have an important adjunctive role in addressing the former (NCT01439841).

Probiotics have been safely administered to subjects with HIV, have alleviated GI symptoms associated with viral replication, have proven active against bacterial vaginosis in HIV-infected women, and have enhanced growth in infants with congenitally acquired infection (6). However, the effects of probiotics on the mucosal immune environment in HIV-infected patients are currently unknown.

In this issue of the JCI, Klatt et al. demonstrate that the addition of a prebiotic/probiotic mixture to ART enhances mucosal immunity in SIV-infected pigtail macaques by reconstituting GI CD4 $4^{+} \mathrm{T}$ cells and decreasing inflammation-associated fibro- sis in the gut-associated lymphoid tissue (GALT) (7). The results are intriguing and expand our perspective on the potential impact of probiotics in HIV infection.

But is this reconstitution clinically important? As the case illustrates, many patients infected with HIV are not considered to be at average risk for colorectal cancer. HIV-infected patients have been shown to have a higher prevalence of colon cancer, to develop adenocarcinomas at a younger age, and to develop more aggressive lesions compared with HIVuninfected controls (8). They also have a predilection toward developing right-sided adenomas that may escape colonoscopic detection and result in delays in diagnosis and care. The increased prevalence of GI cancer in HIV is not limited to the colon; recent data suggest that patients with AIDS are at increased risk for esophageal and gastric carcinoma as well (9). These features may be due to a lack of appropriate host immune surveillance in the gut, increased intestinal inflammation and reactive oxygen species associated with the immunopathogenic effect of HIV, and/or the presence of certain opportunistic enteropathogens. Despite these findings, national and societal guidelines do not currently recommend earlier or more frequent screening colonoscopies in HIV-infected patients (10).

One could imagine a scenario whereby reconstitution of the GI $\mathrm{CD}^{+}$compartment with probiotics could enhance immune surveillance, promote cancer cell apoptosis, and in some capacity, restore the colonic mucosa to its native pre-HIV state. The authors imply that one of the mechanisms behind GI immune reconstitution with probiotic administration is a reduction in inflammation-associated fibrosis within the lymphoid follicles of the GI tract. Perhaps probiotics also reduce the amount of oxidative stress on the colon in the same manner, a feature of colitis-associated carcinogenesis that may partially explain the increased preva- 

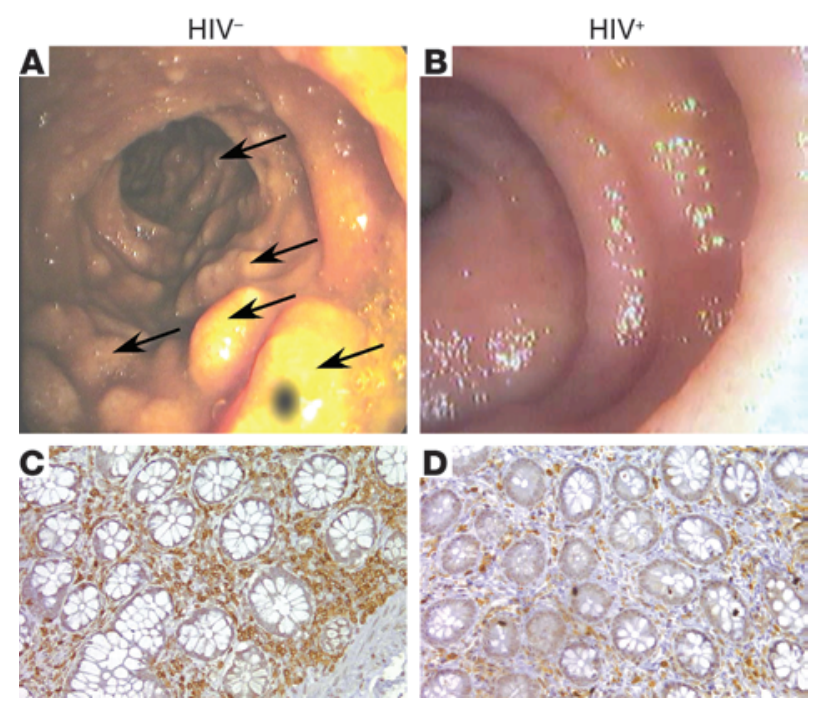

\section{Figure 1}

Endoscopic and histological analysis of the terminal ileum in an HIV-uninfected $(\mathbf{A})$ and HIV-infected patient (B). Immunohistological staining for CD4 was performed by sectioning biopsies from ileal lymphoid tissue followed by H\&E staining and immunostaining for CD4 (C and D). Reproduced with permission from The Journal of Experimental Medicine (2).

lence of colon cancer in HIV patients. The use of probiotics may be an inexpensive, noninvasive, and generally well-tolerated adjunctive treatment for HIV, should further research support this indication.

\section{Acknowledgments}

The authors thank Jason Brenchley and Daniel Douek for permission to use Figure 1.

Address correspondence to: Judith A. Aberg, Director of Virology, Bellevue Hospital Center, Director, Division of Infectious Diseases and Immunology, NYU School of Medicine AIDS Clinical Trials Unit, 550 First Ave., BCD 5, Room 558,
New York, New York 10016, USA. Phone: 212.263.6565; Fax: 212.263.8264; E-mail: judith.aberg@nyumc.org.

1. Ciorba MA. A Gastroenterologist's guide to probiotics. Clin Gastroenterol Hepatol. 2012;10(9):960-968.

2. Brenchley JM, et al. CD4+ T cell depletion during all stages of HIV disease occurs predominantly in the gastrointestinal tract.J Exp Med. 2004;200(6):749-759.

3. Cunningham-Rundles S, et al. Effect of probiotic bacteria on microbial host defense, growth, and immune function in human immunodeficiency virus type-1 infection. Nutrients. 2011;3(12):1042-1070.

4. Brenchley JM, et al. Microbial translocation is a cause of systemic immune activation in chronic HIV infection. Nat Med. 2006;12(12):1365-1371.

5. Chun T-W, et al. Persistence of HIV in gut-associated lymphoid tissue despite long-term antiretroviral therapy. J Infect Dis. 2008;197(5):714-720.
6. Wilson NL, D Moneyham L, Alexandrov AW. A systematic review of probiotics as a potential intervention to restore gut health in HIV infection [published online ahead of print July 31, 2012]. J Assoc Nurses AIDS Care. doi:10.1016/j.jana.2012.04.004.

7. Klatt NR, et al. Probiotic/prebiotic supplementation of antiretrovirals improves gastrointestinal immunity in SIV-infected macaques. J Clin Invest. 2013;123(2):903-907.

8. Bini EJ, Green B, Poles MA. Screening colonoscopy for the detection of neoplastic lesions in asymptomatic HIV-infected subjects. Gut. 2009;58(8):1129-1134.

9. Persson EC, Shiels MS, Dawsey SM, Bhatia K, Anderson LA, Engels EA. Increased risk of stomach and esophageal malignancies in people with AIDS. Gastroenterology. 2012;143(4):943-950.

10. U.S. Preventive Services Task Force. Screening for colorectal cancer: U.S. Preventive Services Task Force recommendation statement. Ann Intern Med. 2008;149(9):627-637. 